\title{
Generation of Virtual Point Source by Diffraction from Screen Edge as an X-ray Source for High-resolution Holography
}

\author{
$\underline{\text { Yoshio Suzuki }}^{1,2,3^{*}}$, Akihisa Takeuchi ${ }^{4}$
}

1. Graduate School of Frontier Science, University of Tokyo, Kashiwa, Chiba 277-8561, Japan

2. Photon Factory, IMS/KEK, Tsukuba, Ibaraki 305-0801, Japan

3. Toyama Co. Ltd, Yamakita, Kanagawa 258-0112, Japan

4. Japan Synchrotron Radiation Research Institute (JASRI), Spring-8, Sayo, Hyogo 679-5198, Japan

* Corresponding author, yoshio2081@gmail.com

X-ray holography [1] is one of the promising methods for high-resolution phase-contrast imaging. The spatial resolution of traditional holography, Gabor holography with spherical wave and Fourier transform holography with point reference source, is essentially determined by the source size of spherical wave. The small x-ray reference sources are now usually made with modern X-ray focusing optics such as Fresnel zone plates $[2,3]$. An idea for making small virtual $x$-ray source is utilization of external total reflection of very short convex cylindrical mirrors, and the method is already applied to Gabor holography in the hard x-ray region [4]. A crossed waveguide optics [5] is also applied to hard X-ray beam confinement and holographic imaging. Here, we propose a method for generation of small virtual point source by combining two screen-edges, and its application to Gabor type holography is also described.

It is known that the diffracted wave from a screen edge works as an infinitely small secondary source, when the edge is observed from the shadow region of the screen. The edge-diffraction of x-rays was firstly applied to measurement of focused beam profiles in the hard x-ray region [6]. The method is called "darkfield knife-edge scan", where a transparent knife-edge (edge of phase object) is used, and the directly transmitting beam is masked with a beam stop for sensing only the diffracted wave field. As a result, the edge of transparent object can be regarded as a pseudo-slit of very narrow width. This technique is now widely used in the hard x-ray region, and sub-ten nanometer beam size has been directly measured [7]. These results indicate that the effective slit width much less than ten nanometers is realized by the darkfield knife-edge method. It is easily speculated that the high-resolution imaging would be capable by applying the edge-diffracted wave to the virtual point source for holographic imaging. The optical system for the Gabor holography with virtual point source by the edge-diffraction is schematically shown in Fig. 1. Two straight knife-edges are set in an orthogonal configuration to generate the point-like secondary source. The crossed knife-edge is illuminated with a collimated x-ray beam, and the object is placed in the spherical wave field diffracted from the crossed knife-edge. The propagating wave through the object is detected with an X-ray imaging detector. A beam stop is placed between the crossed edge and imaging detector to suppress halo due to the intense direct beam.

The experiment has been carried out at the first experimental station of Beamline 20XU, Spring-8. X-ray energy of $9.85 \mathrm{keV}$ (wavelength of $1.259 \AA$ ) is selected with a Si 111 double crystal monochromator. The first experimental hutch is located at about $80 \mathrm{~m}$ from source point. The x-ray beam is condensed with a high-efficiency kinoform Fresnel zone plate [8], and an order-selecting-aperture with a diameter of $20 \mu \mathrm{m}$ is placed near the focus (not shown in the figure). The projection image is taken with a CCD-based image sensor coupled with $\mathrm{Gd}_{2} \mathrm{O}_{2} \mathrm{~S}: \mathrm{Tb}^{+}$scintillator screen. The CCD has 1024 x 1024-pixel format, and the effective pixel size was $6.5 \mu \mathrm{m}$ in the experiment. The knife-edge used in the experiment is gold wire with a diameter of $25 \mu \mathrm{m}$. The two wires are contacted to each other to minimize the astigmatism due to 
separation of vertical and horizontal source positions. The sample is resolution test patterns made of 0.5 $\mu \mathrm{m}$-thick tantalum deposited on a SiN membrane. The sample was set at about $10 \mathrm{~mm}$ from the virtual source point, and the imaging detector was placed at $2.2 \mathrm{~m}$ from the source. Therefore, the geometrical magnification of hologram was 220 . Then, the effective pixel size of reconstructed image is $30 \mathrm{~nm}$. The beam stop is crossed-blade made of thick tantalum plate that was placed at about $0.2 \mathrm{~m}$ from the sample position.

A typical result on Gabor holography is shown in Fig. 2. Figure 2(a) shows the measured hologram, and the numerically reconstructed phase-contrast image is shown in Fig. 2(b). The fine structures of the object are clearly reconstructed. The theoretical spatial resolution of reconstructed image is simply determined as $\lambda / N A$ in the case of periodic structure [3], where $\lambda$ is wavelength, and $N A$ is numerical aperture of hologram defined by $\sin \theta$. The $\theta$ is half acceptance angle of hologram. In the present experiment, the full acceptance of hologram is about $2.6 \mathrm{mrad}$, as shown in Fig. 2(a). Therefore, the theoretical resolution limit is calculated to be $100 \mathrm{~nm}$. The spatial resolution of reconstructed image was evaluated from the sharpness of edge structure. The resolution defined by $25 \%-75 \%$ width of edge broadening is $90 \mathrm{~nm}$ that is consistent with the theoretical value.

The spatial resolution of holographic imaging is not restricted by the performance of optical elements. Therefore, in principle, there are no limitations on spatial resolution, except for the noise limitation due to the limited photon flux density. In the present experiment, the incident beam was not fully coherent. When a sufficiently coherent $\mathrm{x}$-ray beam is used, it is possible to perform the Fourier transform holography by placing the object and virtual source at the same distance. This would provide holographic imaging free from twin image overlap [9].

[1] S. Aoki and S. Kikuta, Jpn. J. Appl. Phys. 13 (1974) 1385.

[2] I. McNulty, et al., Science 238 (1992) 1009.

[3] Y. Suzuki and A. Takeuchi, Jpn. J. Appl. Phys. 51 (2012) 086701.

[4] S. Aoki, et al., Jpn. J. Appl. Phys. 44 (2005) 417.

[5] S. P. Krüger, et al., Opt. Exp. 18 (2010) 13492.

[6] Y. Suzuki, et al., Jpn. J. Appl. Phys. 44 (2005) 1994.

[7] H. Mimura, et al., Nature Photonics 6 (2010) 112.

[8] A. Takeuchi, et al., Jpn. J. Appl. Phys. 51 (2012) 022502.

[9] The experiment has been carried out wit

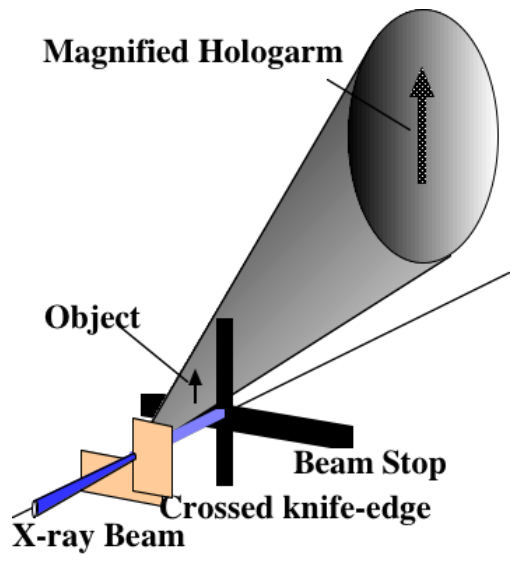

Figure. 1. Schematic diagram of optical system for Gabor holography.
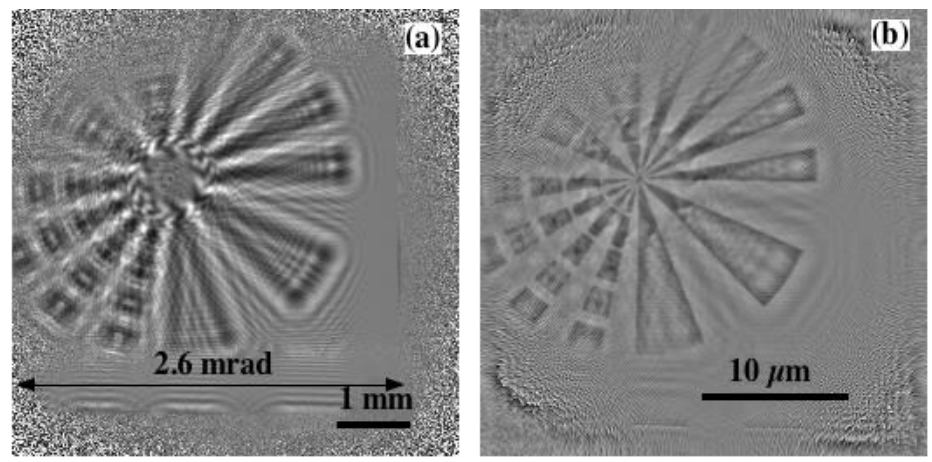

Figure. 2. (a) measured hologram and (b) reconstructed phase image. The full angular size of hologram is $2.6 \mathrm{mrad}$ as shown in (a). Exposure time is $10 \mathrm{~s}$. Flat field correction was done using a blank image without object. 